\title{
PREVENTIVE PLANNING MODEL FOR RESCUE PRIORITY MANAGEMENT IN SEISMIC EMERGENCY
}

\author{
MONICA CARDARILLI, MARA LOMBARDI \& MASSIMO GUARASCIO \\ Department of Chemical Materials Environmental Engineering, Sapienza University of Rome, Italy.
}

\begin{abstract}
This study stems from the detection analysis of damaged buildings in Nocera Umbra (Central Italy), which has been affected by the earthquake sequence during 1997-1998, causing serious damages to material goods and human casualties. This natural disaster highlighted the need to achieve a strategic planning during the emergency phase based not only on effective actions but also on the efficient management of rescue and relief operations.

The application of Quantitative Risk Analysis (QRA) has led to the seismic risk assessment for estimating the potential level of the expected damage, on heritage buildings and human lives.

Data and information have been gathered during the surveys carried out after the major seismic event. A deep analysis has highlighted the existence of a correlation ratio between the critical structural features and the propensity of buildings to collapse. Considering either the exposed population, this information represents the crucial element for a relief management plan.

All risk indicators associated to each damage scenario have allowed to assign a scale of priorities at every relief operations for an effective risk mitigation based on damage level and expected casualties.

The ex-post emergency organization would be able to make more efficient both allocation and management of resources, equipment and technical teams during rescue activities. This is strongly connected to the ex-ante priority planning of the operations related to magnitude and distribution of expected collapses and casualties thank to the simulation performed to the potential risk scenarios. Keywords: building collapse, Quantitative Risk Analysis, seismic emergency, vulnerability index
\end{abstract}

\section{INTRODUCTION}

Italy is a Country with high seismicity, due to the frequency and strength of the earthquakes that have affected its territory and we know the effects thanks to the written testimonies left by many eyewitnesses over the centuries that have allowed us to reconstruct the earthquake [1]. Being a Country subjects to earthquake, quantitative risk measurement is an imperative necessity for optimum emergency management and for the controlled planning of costs for the prevention and protection of individuals and cities [2]. The earthquake manifests itself as a rapid and violent ground shake and occurs unexpectedly without notice. It is very hard, but its consequences, in terms of victims, material damage and population involved, are often dramatic.

An earthquake is characterized by a sequence of shocks called seismic period, which sometimes precedes and almost always follows the main shock. The oscillations caused by the passage of seismic waves cause horizontal impetus on the constructions, causing serious damage or even collapse if they are not constructed with anti-seismic criteria.

In addition, the shaking of buildings is not always the same, but it depends on the local conditions of the area, in particular the type of land on the surface and the shape of the landscape [3].

For the evaluation and management of territorial risk due to the earthquake, the methodology of Quantitative Risk Analysis (QRA) has been applied which enables an integrated multidisciplinary analysis of the factors that contribute to the assessment of the risk in the territory of the municipality of Nocera Umbra. Seismic risk, so that it may be addressed and managed at best, needs to be studied and analyzed through contributions from a variety of 
disciplines and sectors such as seismology, geophysics, geomatics, structural engineering, and emergency.

\section{GEOLOGICAL SETTING AND SEISMIC ACTIVITY}

Nocera Umbra is a municipality in the province of Perugia. The town is located in the centre of a vast mountainous area, along Via Consolare Flaminia, which connects the towns of Foligno and Gualdo Tadino. Located on the top of a rock spur, dominated by the small River Topino and the Flaminia, Nocera Umbra, with its perched appearance, shows clear signs of its past defensive structure.

Nocera Umbra is characterized by the expanse of lithological formations ranging from Red Scaglia to Marnoso-Arenacea, attributed to the Appennines series.

The latter shows the alternation of sedimentary rocks (limestone, marl, clayey and marble limestone), each with different lithological and mechanical characteristics, above which there is a layer of heterogeneous soil with poor mechanical properties. There are therefore differently erodible soils.

Seismic Microzonation evaluates site local effects as those effects that involve changes in the seismic wave propagation (amplification or reduction) in the more superficial layers of soil. Seismic Microzonation studies allow to characterize the territory by identifying and delimiting areas with homogeneous behaviour, distinguishing between stable zones, stable zones Susceptible to local amplification and areas subject to instability. For Nocera Umbra, the amplification zones have been obtained using the map of the amplification zones, as illustrated below.

In the present study, we will use the FA amplification factors from the detailed microsynculation investigations carried out [4].

Started in September 1997, the earthquake struck the fascination of the Apennine chain between Marche and Umbria, already home to destructive earthquakes.

The intensity of the strongest shock (5.8 Richter - IX Mercalli) is in line with those of the historical earthquakes that have affected East Umbria in the past.

\section{SEISMIC RISK ASSESSMENT}

Italy is one of the most seismic countries in the Mediterranean because of the frequency of earthquakes that have historically affected its territory and the intensity that some of them have achieved, resulting in a significant social and economic impact.

The seismic risk assessment for Nocera Umbra was conducted using the QRA methodology, in particular by using the ETA (Event Tree Analysis).

This analysis, in fact, is based on a logical-intuitive structure. In addition, since the succession of steps describing the evolution of the danger flow is chronological, it is easy to traverse the chain of events that derive from an initiating event and reproduce the flow itself. It is in fact, as a sequential and interconnected representation of all possible alternative paths of evolution of danger scenarios triggered by a seismic event. Each event consequence is associated with a conditional probability from all previous events in the branch [5].

\subsection{Initiator event probability}

The knowledge of the Italian seismicity is made possible by the great number of studies and documents about the effects that earthquakes have provoked in the past in the various geographical areas. Especially in recent years, these studies have been used in regional analysis 
for zoning (seismic classification) or Microzonation. In the latter case, the assessment of the hazard involves the identification of areas which may, during a seismic shock, be subject to amplification phenomena.

The likelihood of occurrence associated with the initiating event may be evaluated from the probabilistic maps of the seismic hazard of the national territory, expressed in different shaking parameters on a square mesh grid of approximately $5 \mathrm{~km}$ per side, provided by the INGV (National Institute of Geophysics and Vulcanology). The maps used for the present work report the maximum horizontal acceleration of the ground as defined by [6] as the shake parameter, corresponding to PGA.

These maps have been calculated for different probability of overcoming in 50 years. For each estimate, we get the 50th percentile distribution (median map, which is the reference map for any probability of overcoming). Thus, Hazard Curves have been reconstructed showing the PGA trend for the various annual overcoming probabilities needed to determine the likelihood of occurrence of the Initiator Event $(\mathrm{P}(\mathrm{EI}))$.

By constructing a semi-logarithmic graph that has ascending times and ordered PGA thresholds, it is possible to determine the return time, starting with the PGA value of the event of September 26th, 1997 for Nocera Umbra, obtained using the Attenuation Law [7], which was derived from a European database consisting of 422 recordings generated by 157 earthquakes measuring magnitude Ms between 4.0 and 7.9, and a focal depth of less than $30 \mathrm{~km}$.

$$
\log (P G A)=-1.48+0.266 M-0.922 \log r+0.117 S_{A}+0.124 S_{S} \pm 0.25
$$

where:

$\mathrm{M}=$ registered Magnitude

$r=\left(d^{2}+3.5^{2}\right)^{0.5}$

$\mathrm{d}=$ focal depth (i.e. the distance between the Epicenter and the Hypocenter)

In the semi-logarithmic chart below, the ascending times are reported in relation to the different probabilities and the PGA thresholds for the 50th percentile are ordered.

From the intersection with the interpolation line, the corresponding return time was obtained (See Fig. 1).

Earthquake interception times follow the statistical distribution of a Poisson process. This means that each seismic event in an area is independent in time and space from previous events in the same area and does not affect spatial location, time of occurrence, and size

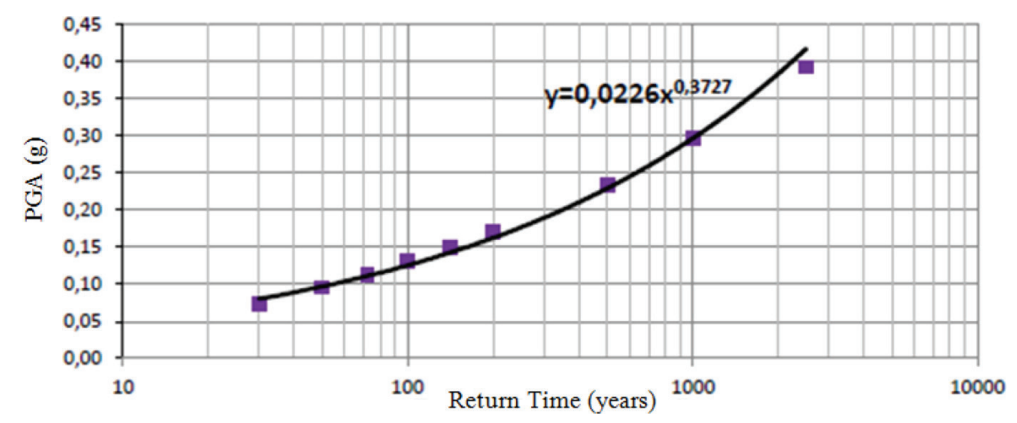

Figure 1: 50th percentile curve. 
(Magnitude) of subsequent ones. Thus, the probability of the Initiator Event is given by the following equation:

$$
\mathrm{P}(E I)=\frac{(\lambda t)^{n}}{n !} e^{-\lambda t}=1.06 * 10^{-2}\left[\frac{\text { events }}{\text { year }}\right]
$$

Initiator Events are the starting point from which the Event Tree is drawn and represent the events that precede or start the chains of subsequent events. Starting from them, we identify the different scenarios of damage that depend on the severity of the consequences.

\subsection{Propensity to collapse}

The first Tree node represents the probability of the collapse event occurring for a given building. This is affected by several variables, the structural vulnerability of the building itself and the amplification of seismic shock, determined by the morphology and geological characteristics of the soil.

The estimation of structural vulnerability is a necessary step to understand the response of a building to seismic stress, that is, its propensity to damage in case of an earthquake [8].

The sample of examined buildings consists of 150 buildings, all of them masonry, located in Nocera Umbra. The choice of the sample was dictated by the desire to treat together buildings of the same type of construction and the fact that masonry buildings, both ordinary and monumental historical value, are one of the most vulnerable artefacts of the earthquake [9].

For the calculation of the vulnerability of the sample examined, the data in the 1st level detection, damage and promptness data for ordinary buildings in post-seismic emergency (AeDES Forms) were used. In activities that characterize the post-earthquake, a significant moment is usually represented by damage relief and the assessment of seismic agility. Post-seismic emergency agility assessment is a temporary and expeditious evaluation formulated on the basis of an expert judgment and conducted in a limited time. It is based on simple visual analysis and the collection of easily accessible information to determine whether buildings affected by the earthquake may be used while reasonably protected human life. AeDES Forms therefore quantify the damage reported by each building on the basis of the outcome of the assessment.

\subsubsection{Building Features and Numeric Encoding}

In order to arrive at the determination of the probability of collapse for each of the buildings under study, several different characteristic factors must be considered for each of them. The parameters assumed at the base of the vulnerability study were identified as more important factors for the description of the seismic behaviour of masonry buildings:

- Number of floors;

- Regularity of shape;

- Period of construction;

- Position in the aggregate.

The first two give information about building form and size, while the second ones about resistance and stability characteristics.

The height of the building has a significant influence on the resistance of the structures under the action of seismic motions. In fact, it plays a significant role under the effect of seismic stresses [10]. 
Regularity in plan and height is an essential requirement for defining the seismic response of a building. An uniform distribution of strength and stiffness along the height of the building is a significant factor for the good seismic behaviour of the structure. Regularity in fact avoids the development of asymmetries and hence the formation of plastic hinges (weak points) resulting in an uneven concentration of agent stresses [11].

Construction period parameter takes into account existing regulations at the time of construction and therefore the construction techniques and the anti-seismic technologies used. For this reason, it was considered appropriate to take into account the main technical legislations for masonry buildings [12, 13].

Finally, it is also important to identify the location of the building within the structural aggregate: isolated, internal, at corner, at edge for considering possible interactions from structural contiguity between adjacent buildings (hammering phenomena).

Each property of the building has been divided into three classes and each one has been assigned to a category (red, yellow, green). The categories were attributed to the contribution of the individual class to the overall structural integrity of the building.

Using the values of post-earthquake damage reported on the AeDES Forms, a numerical conversion was performed by assigning to each of them a coding ' 0 ' no collapse, and ' 1 ' collapse, in order to achieve a dichotomous classification of the damage reported by the buildings, as below:

- Encoding '0' - building does not collapse:

- null;

- slight damage;

- average damage.

- Encoding ' 1 ' - building collapses:

- serious damage;

- very serious damages;

- destroyed.

The correlation coefficients for the four parameters, previously standardized, were calculated. A correlation analysis was carried out between all the variables considered.

The variance-covariance matrix was calculated, which coincides with the matrix of correlation in our case. It is possible thus to evaluate how much a pair of parameters are correlated, and the sign indicates whether the correlation is positive or negative.

The major correlation is between the position and the construction era, which are the parameters that provide information on the building's resistance. In particular, it turns out that the most recent buildings are in a corner or end position in the aggregate.

A good correlation is also between the building age and the number of plans: that is what it is indicating that most recent buildings have more floors.

Table 1: Correlation matrix.

\begin{tabular}{lllll}
\hline & Position & n. Floors & Age & Regularity \\
\hline Position & 1 & 0.016 & 0.431 & 0.101 \\
n. Floors & 0.016 & 1 & 0.164 & 0.028 \\
Age & 0.431 & 0.164 & 1 & 0.024 \\
Regularity & 0.101 & 0.028 & 0.024 & 1 \\
\hline
\end{tabular}


One further consideration that emerges is that recent buildings are also the most irregular.

To determine the weights to associate with each parameter, two different approaches have been used:

1. Principal Components Analysis (PCA);

2. Normalized-Correlation with 'Collapse - does Not Collapse (C/NC)' variable.

\subsubsection{Correlation analysis of collapsing}

From the variance-covariance matrix, which coincides with the correlation matrix in the case of standardized variables, the main self-extractor is extracted to highlight the covariates between the variables considered. This was obtained by the factorial analysis method performed with the data processing software R.

The main driver co-ordinates represent the weights of the coded parameters. The positive weight indicates that the coarse reference parameter in the same sense with the other three, that is, the combined effect of the interactions and covariation is concordant. Another weighing system is the normalized correlation coefficients of each single parameter with the standard collapse/no-collapse variable.

The obtained variable was correlated with the four characteristic parameters of the buildings.

The parameters were taken individually to study how each of them influences propensity to collapse. From the weight analysis of the correlation coefficients, it may be seen first that they are all differentiated between them and with not very high values.

The position is the most linked feature of the propensity to collapse, while the number of floors is the least-related parameter. This is justified by the fact that the buildings, being in masonry, do not have a large variation of the floors parameter: most of it consists of two, three or four floors. So, as far as brick buildings are concerned, the collapse will be associated with other variables.

In order to have a better visualization of the weights thus obtained, the percentages of buildings for the individual parameter encodings for the collapse/no-collapse variable have been calculated. The relative percentages of collapsed buildings were also evaluated.

\subsubsection{Evaluation of the Vulnerability Index}

Thus, with both the methodologies, the Vulnerability Index (Iv) for each building was calculated by adding the products between the weights obtained and the standardized encoded values of the building parameters. It is denoted that both calculated Iv have a percentage of $50 \%$ explained. Since the apparent capability of an Iv range has not been evident from the results of the calculated vulnerability indices, the graph has been comparing the two Indices thus obtained and returning the number of coincident values for each identified coordinate.

The value of the Iv, valued by correlation coefficients, is higher than the equivalence condition represented by the other one.

\subsubsection{Regression model}

Through the use of regression models, it was possible to extrapolate the value of the propensity to collapse, starting with the calculation of the vulnerability, using the data reported on the damage relief cards. To get the propensity to collapse from the estimated values of vulnerability and the collapse/no-collapse variable, the regression models have been applied. 
The application of regression models to buildings surveyed with damage relief forms allows to estimate the probability of collapse depending on the vulnerability values and site amplification.

To analyze a dichotomous variable, we use Logistic regression, which represents a Generalized Linear Model (GLM) in which the link function is the 'logit' of the probability of belonging to a category (code ' 1 ') with respect to not belonging to it (code ' 0 ').

The propensity to collapse is then defined by the following equation:

$$
\theta=\mathrm{P}(Y=1)=\frac{\mathrm{e}^{\left(\beta_{o}+\beta_{1} x_{1}+\beta_{n} x_{n}\right)}}{1+\mathrm{e}^{\left(\beta_{o}+\beta_{1} x_{1}+\beta_{n} x_{n}\right)}}
$$

where $\mathrm{x}_{\mathrm{i}}$ represents the building vulnerability and local amplification, and $\beta$ the regression coefficients.

The statistical significance (p-value) of the regression coefficients obtained from the first processing is higher than the respective value reported by the regression of collapse/nocollapse variable where both fall within the limit value of 0.05 .

The second elaboration of the vulnerability estimated by correlation coefficients shows a significant increase in the statistical significance of the variable, hence the ability of the variable to explain the propensity to collapse.

The AIC index allows to evaluate the adaptation of data with the link used. The AIC indicator value from both outputs indicates a discrete adaption of the logit model to the data. The AIC of the second implementation also shows an improvement in model adaptation to data compared to the previous case.

The Iv model provides a p-value and a lower AIC, hence a greater statistical significance of the performed estimate and better model quality than the data implemented.

It may be observed that the highest values that characterize the range of the estimated vulnerability with the correlation coefficients are also reflected in its propensity to collapse. In particular, the range of the right table is also characterized by greater explanatory capacity, with a level of explanation of $61 \%$ versus $44 \%$ for the two different implemented Iv.

Since the Factor of Amplification plays an important effect, this is reflected in the value of the collapse probability, in fact the propensity values are increased in the minimum and maximum value of the range.

Based on the results of regression, it is considered that the most effective Iv in explaining collapses is the one resulting from second processing, as expected.

Regression models therefore allow for an estimate of the propensity to collapse that takes into account local seismic microzonation by providing an assessment of the most representative buildings and hence a more effective preventive action and/or more aware emergency management in the post-earthquake.

Table 2: Propension to collapse

\begin{tabular}{llll}
\hline & \multicolumn{2}{l}{ Vulnerability } & with Amplification \\
\cline { 2 - 4 } & PCA & C/NC & C/NC \\
\hline $\min$ & 0.07 & 0.08 & 0.33 \\
$\max$ & 0.51 & 0.69 & 0.93 \\
\hline
\end{tabular}




\subsection{Salvability estimation}

In the second and third bifurcations, crowding and exposure are considered. The second Tree node represents the exposure, expressed in this specific case in terms of human lives, as for civil protection purposes it is necessary to estimate the social losses expected as a result of an earthquake [14].

The starting data required for the quantification of the exposed to a given seismic event is that of the population resident in the analyzed buildings [15]. The population per building varies considerably depending on the location and is a function of the characteristics and dimensions of the buildings. To determine this parameter may be used the data provided by the ISTAT Census or the data reported on the AeDES Forms where available, as in our case. From the AeDES Forms, the number of occupants for each building was estimated by the exhibitors.

The earthquake event timetable is a determining factor for the estimation of the victims, as it affects the number of people present inside the buildings at the time of collapse [16].

In the construction of Events Tree must be considered two scenarios: daily and nightly by using Coburn model [17] applied to residential buildings.

The mediator most likely to influence the likelihood of an exposed individual being involved in the collapse is represented by the building and its vulnerability. In fact, injuries caused by the involved are caused by the size and mode of collapse of the building, depending on the type of structure, intensity and type of construction shock [18].

In the Coburn model, the parameters to be considered for determining the number of victims among the Exposed (E) people, in the case of masonry structures such as those in the present study, are as follows:

- Trapped (I): $60 \% * \mathrm{E}$

- Victims (V): $20 \% * \mathrm{I}$

By applying these reductive factors, the number of deaths associated with the earthquake is obtained according to the scenario assumed, which is indispensable for the quantification of the damage, both expressed in terms of human lives that in economic terms associated with one or more seismic events analyzed.

The second mediator affecting the salvability of these individuals is the management of relief, in fact, the lengthening of survival research times greatly exacerbates the likelihood of getting them out of life again [19].

In the construction of the Event Tree, a parameter is used that takes into account the speed of the intervention of the rescuers, assuming that it takes place within the first $48 \mathrm{~h}$ of the earthquake, beyond which the probability of survival is reduced to values below $30 \%$.

\subsection{Risk indicators and acceptability thresholds}

In the last bifurcation is considered the efficiency indicator of emergency management that takes into account the rescue and self-relieving (salvability).

From Event Tree Analysis, it is possible to determine the probabilities of all possible endof-turn scenarios [20]. From the knowledge of the latter, the expected value for the scenario considered was the number of expected casualties [21].

Afterwards, the retrospective distribution was determined, which provides information on the social perception of risk (Social Risk). 
Social risk perception may be expressed in terms of the Social Damage Curve, where the probability of overcoming the corresponding threshold in the order of $\mathrm{P}$ is associated with each possible degree of damage expressing the number of expected casualties $\mathrm{N}$.

In order to develop prevention and mitigation policies, it was considered necessary to make the assessment scenario more complete by comparing the expected Risk Indicator with an appropriate limit value of the trend of the retrofitted distribution by means of curves representing tolerance limits considered socially acceptable or not acceptable.

The red line represents the threshold of unacceptability, while the green line is the threshold of acceptability.

Analyzing the graph above, we may see that the distribution of the retrofitting is completely within the threshold, although its performance is close to the straight line unacceptability.

It should be noted that the levels of acceptability of the cumulative risk used in the present study are those related to railway tunnels [22], which provide maximum values of acceptability and unacceptability of probabilities equal to $10^{-4}$ and $10^{-2}$.

The area underneath this curve represents the Expected Value $\mathrm{E}(\mathrm{N})$, the Risk Indicator as sum of the number of casualties expected for the respective scenario.

From the graphic representation below, we may see the probability of achievement and/or overcoming the expected number of victims for a given PGA value (See Fig. 2).

\subsection{Mosaic model}

For a rational and effective emergency management in a seismic event, it is necessary first to subdivide the whole urban grid into sub-areas, in order to associate one or more of them with each rescue team through the application of the 'Mosaic Model'. The territory on which buildings fall into is to be divided into 'tiles', or irregular polygons delimited each by urban road. The tiles at first are defined according to the roads deemed feasible in the event of an emergency.

In the specific case, having analyzed a small number of buildings, there were areas of intervention containing inside a comparable number of buildings.

The tiles, uniquely defined with increasing numbering, are the basic element on which to carry out estimates of the quantification of the victims and the injured in order to order the tiles on the intervention scale and associate them with the rescue teams [23]. Sending human resources and equipment is a priority in those cards that present more trapped and injured.

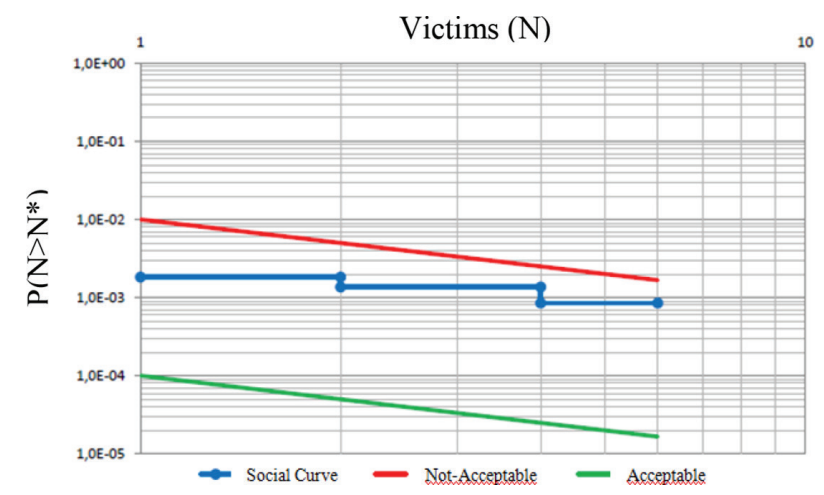

Figure 2: Social damage curve with acceptability thresholds (for a type building). 
Adding the wounded from the Event Tree for the buildings belonging to the same tile, the number of injuries was determined for each of them. In this way, it was possible to determine the intervention order of the rescue teams, starting from the tile with the most injured to the one with the least number (See Fig. 3). Tiles play a key role in organizing relief, estimating the number of injuries expected and sending the number of intervention teams needed. With the QGIS software, the tiles and their associated buildings were represented.

Other information that may be given to the tiles in addition to the number of wounded is the probability of collapse associated with each building for a previously assessed PGA date. This would provide useful guidance to rescue teams already in the early hours of the event, during which the rescuers work uninterruptedly to help the trapped potentially.

The evaluations were carried out for the most critical tile since it is characterized by buildings with maximum and minimum probability values, for the case study, and because it is ranked first in the ranking for the intervention order and therefore by the number of inhabitants.

The maximum and minimum trend of the tile has also been analyzed as a probability of overcoming the back-cumulated curve (See Fig. 4). In this case, the scenario of damage is represented by the collapse of buildings. The damage variable is between 0 (no building collapses) and 10 (collapses all buildings).

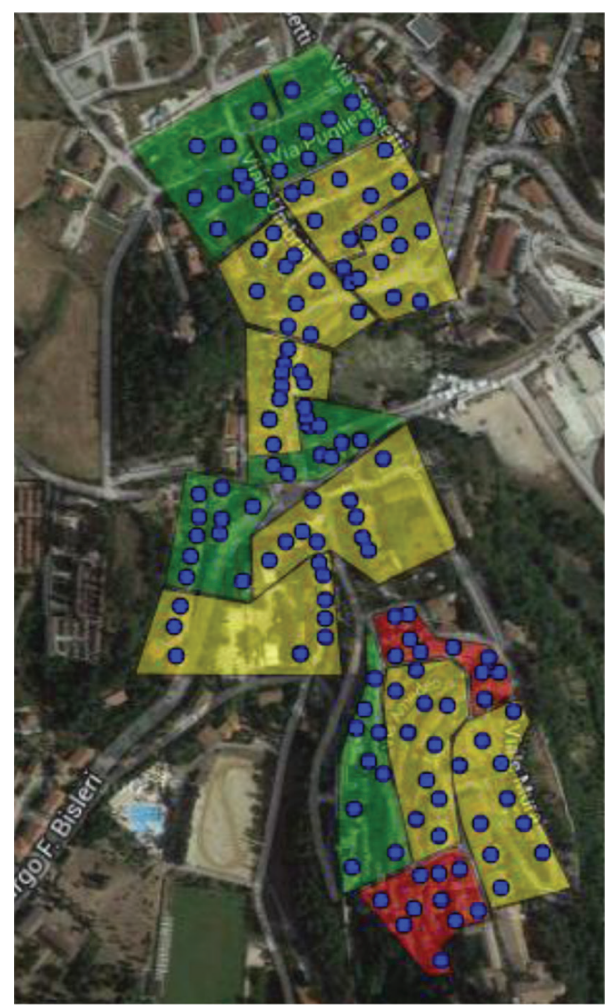

Figure 3: Intervention order for each tile. 


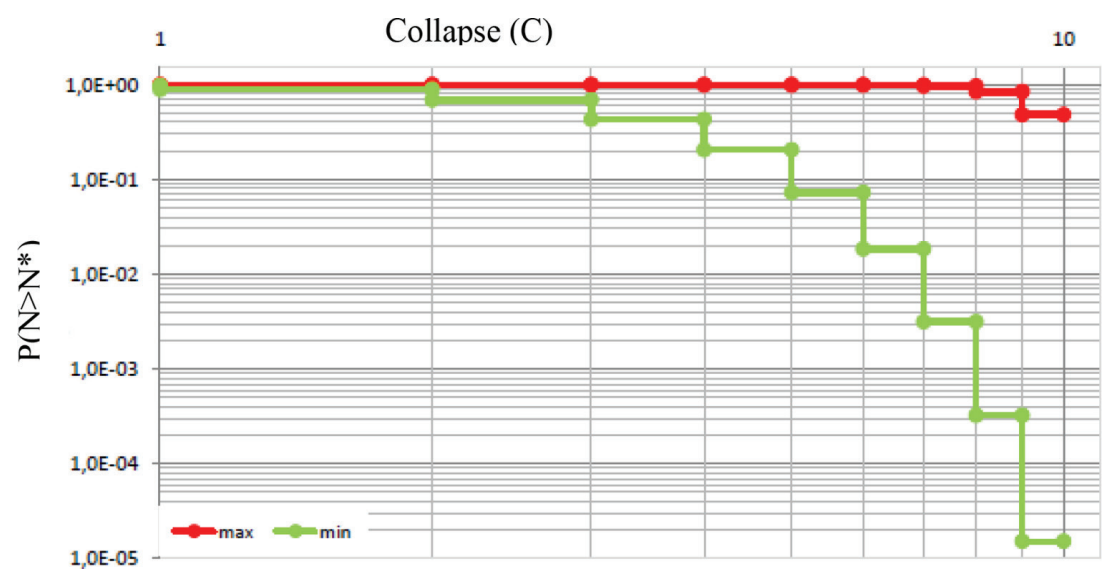

Figure 4: Maximum and minimum Social Damage Curves of the most critical tile.

\section{CONCLUSION}

The work involved the proposal of a methodology useful for the prevention, planning and management of the seismic emergency for the territory of the Municipality of Nocera Umbra. The analysis was carried out according to Events Tree that allows to determine collective and individual Risk Indicators based on the expected number of victims. Risk indicators have been evaluated either by referring to the victims or by the collapse of each mosaic 'tile' in the city itself: in the first case, the areas at greater risk within the urban area; in the second allows to compare the level of risk of different buildings.

The indicators would then allow for any intervention priorities for relief to reduce seismic risk. Inside each tile, in fact, the buildings that could have the largest number of victims were identified. It will be exactly those in which the team will intervene at the first moment of the emergency.

The data thus obtained, collected in the form of databases, represent an indispensable tool for timely and effective seismic emergency management.

The application of the described model and the 'mosaic' approach to an entire urban nucleus would allow for a planning of the emergency that is capable of first dimensionizing the resources needed to manage the post-earthquake at the variation of the magnitude of the shock.

Anyway for a more comprehensive and exhaustive analysis, if good quality data is available, other parameters such as degree of apprehension, height interception, site morphology etc. should be considered.

\section{REFERENCES}

[1] Ariki, F., Shima, S. \& Midorikawa, S., Earthquake disaster prevention of Yokohama City. Journal of Japan Association for Earthquake Engineering, 4(3), pp. 148-153, 2004. https://doi.org/10.5610/jaee.4.3_148

[2] Bal, I.E., Crowley, H. \& Pinho, R., Displacement-based earthquake loss assessment for an earthquake scenario in Istanbul. Journal of Earthquake Engineering, 11(2), pp. 12-22, 2008.

https://doi.org/10.1080/13632460802013388 
[3] Erdik, M., Sesetyan, K., Demircioglu, M.B., Hancılar, U. \& Zulfikar, C., Rapid earthquake loss assessment after damaging earthquakes. Soil Dynamics and Earthquake Engineering, 31(2), pp. 247-266, 2011.

https://doi.org/10.1016/j.soildyn.2010.03.009

[4] GNDT/SNN Ratio Seismic Microzonation Project Umbria-Marche Nocera Umbra: Seismic Microzonation Elements, Milan, 1999.

[5] Spence, R.J.S., Coburn, A.W., Sakai, S. \& Pomonis, A., A parameterless scale of seismic intensity for use in seismic risk analysis and vulnerability assessment. In: Society for References Earthquake and Civil Engineering Dynamics (ed.), Earthquake Blast AND Impact: Measurement and Effects of Vibration. Elsevier Applied Science, Amsterdam, 1991.

[6] OPCM (2006) n. 3519

[7] Ambraseys, N.N., Simpson K.A. \& Bommer, J.J., Prediction of horizontal response spectra in Europe. Earthquake Engineering \& Structural Dynamics, 25, pp. 371-400, 1996.

https://doi.org/10.1002/(sici)1096-9845(199604)25:4<371::aid-eqe550>3.3.co;2-1

[8] Calvi, G.M., Pinho, R., Magenes, G., Bommer, J.J., Restrepo-Velez, L.F. \& Crowley, H., The development of seismic vulnerability assessment methodologies over the past 30 years. ISET Journal of Earthquake Technology, 43(4), pp. 75-104, 2006.

[9] Lagomarsino, S. \& Giovinazzi, S., Macroseismic and mechanical models for the fragility and damage assessment of current buildings. Bull Earthquake Engineering, 4, pp. 445-463, 2006. https://doi.org/10.1007/s10518-006-9024-Z

[10] Larionov, V., Frolova, N. \& Ugarov, A., Approaches to fragility evaluation and their application for operative forecast of earthquake consequences. All-Russian conference "Risk- 2000", ed. A. Ragozin, ANKIL, Moscow, pp. 132-135, 2000.

[11] Yeh, C.H., Jean, W.Y. \& Loh, C.H., Damage building assessment for earthquake loss estimation in Taiwan. In: Proceedings of the 12th world conference on earthquake engineering, Auckland, New Zealand, Paper No. 1500, 2000.

[12] Italian Regio Decreto (1937) n. 2105, 22nd November.

[13] Italian Ministerial Decree (1987) n. 141, 9th January.

[14] Bommer, J., Pinho, R. \& Crowley, H., Using a displacement-based approach for earthquake loss estimation. Advances in earthquake engineering for urban risk reduction, eds. S.T. Wasti \& G. Ozcebe, Springer, Dordrecht, 2006.

[15] Crowley, H., Pinho, R. \& Bommer, J.J., A probabilistic displacement-based fragility assessment procedure for earthquake loss estimation. Bulletin of Earthquake Engineering, 2(2), pp. 173-219, 2004.

https://doi.org/10.1080/13632460802013388

[16] Eguchi, R.T., Goltz, J.D., Seligson, H.A., Flores, P.J., Blais, N.C., Heaton, T.H. \& Bortugno, E., Real-time loss estimation as an emergency response decision support system: the EPEDAT. Earthquake Spectra, 13(4), pp. 815-832, 1997. https://doi.org/10.1193/1.1585982

[17] Coburn, A. \& Spence, R., Earthquake Protection, 2nd edn. Wiley, Chichester, 2002.

[18] Spence, R.J.S., Earthquake disaster scenario prediction and loss modeling for urban areas: LESSLOSS report. IUSS Press, Pavia, 2007.

[19] Di Pasquale, G., Ferlito, R., Orsini, G., Papa, F., Pizza, A.G., Van Dyck, J. \& Veneziano, D., Seismic scenarios tools for emergency planning and management. In: Proceedings of the XXIX GAESC, Potsdam, Germany, 2004. 
[20] Jaiswal, K. \& Wald, D., An empirical model for global earthquake fatality estimation. Earthquake Spectra, 26(4), pp. 1017-1037, 2010. https://doi.org/10.1193/1.3480331

[21] Samardjieva, E. \& Badal, J., Estimation of the expected number of casualties caused by strong earthquakes. Bulletin of the Seismological Society of America, 92(6), pp. 2310-2322, 2002. https://doi.org/10.1785/0120010112

[22] Italian M.D. Infrastructures and Transport (2005), Annex 3, Table 4.3.1.

[23] Markus, M., Fiedrich, F., Leebmann, J., Schweier, C. \& Steinle, E., Concept for an integrated disaster management tool. In: Proceedings of the 13th WCEE, Vancouver, BC, Canada, 2004. 